\title{
A ELABORAÇÃO DE MAPAS HIPSOMÉTRICOS NA TOMADA DE DECISÃO EM OBRA, USANDO O SOFTWARE AUTOCAD CIVIL 3D
}

\author{
Francisco Henrique Ximenes da Cruz - henrique.engsan@gmail.com \\ Bacharel em Engenharia Sanitária e Ambiental - Instituição Federal do Ceará - IFCE \\ Fortaleza-Ceará
}

\author{
José Marcos Tavares Pessoa-josemarcostp@hotmail.com* \\ Universidade de Fortaleza - UNIFOR \\ Fortaleza-Ceará
}

\section{Resumo}

Com o advento da tecnologia BIM (Building Information Modelling), um projeto modelado nessa plataforma carrega consigo um conjunto de informações que auxilia em diversos processos na obra. Em obras de infraestruturas, softwares como o AutoCad Civil 3D são primordiais para a tomada de decisão e elaboração do projeto. Em obras de terraplanegem, esses softwares auxiliam na elaborar mapas hipsométricos que através das cores atribuídas as zonas de corte e aterro e suas respectivas quantidades são possíveis determinar a metodologia ideal utilizada e as zonas incisivas para se iniciar a execução do projeto. Além disso, o uso desses mapas em obras de terraplanagem pode gerar uma melhor disposição do canteiro de obra, o que torna a obra mais enxuta. O trabalho terá como objetivo explanar a importância da elaboração desses mapas na tomada de decisões nas obras de infraestrutura. Foi desenvolvido uma análise em um loteamento na cidade de Aquiraz no Ceará para análise da metodologia e apuração de resultados quantitativo do método utilizado.

Palavras-chave: Infraestrutura. AutoCad Civil 3D. Hipsométricos.

\section{THE ELABORATION OF HIPSOMETRIC MAPS IN DECISION- MAKING IN A CONSTRUCTION, USING THE AUTOCAD CIVIL 3D}

\begin{abstract}
:
With the advent of BIM technology (Building Information Modeling), a project modeled on this platform carries with it a set of information that assist in many processes in the work. In infrastructure works, software such as Civil AutoCad 3D is essential for making decision and project design. In earthworks, these software assist in the elaboration of hypsometric maps that through the colors assigned to the cut and fill zones and their respective quantities, it is possible to determine the ideal methodology used and the incisive zones to start the execution. Furthermore, the use of these maps in earthmoving works can generate a better layout of the construction site, which makes the work leaner. The work will aim to explain the importance of preparing these maps in decision-making in infrastructure works. An analysis was carried out in a subdivision in the city of Aquiraz in Ceará to analyze the methodology and determine the quantitative results of the method used.
\end{abstract}

Keywords: Infrastructure. AutoCad Civil 3D. Hipsométric. 


\section{INTRODUÇÃO}

Mapas Hipsométricos são mapas que representam elevações de um terreno por meios da graduação de cores (MELO ET AL, 2017). Esses mapas são utilizados para auxiliar a tomada de decisões em obras e para determinar zonas com variação de elevação. Para isso, segundo RODRIGUES ET AL. (2018) é necessário informações com qualidade e em quantidade suficiente. Devido à essa necessidade surge o BIM (Building Information Modelling) que proporciona informações gerais da obra em um banco de dados para auxiliar a gestão do método construtivo.

A modelagem em BIM carrega consigo informações como o tipo de material a ser utilizado na obra, o modelo digital do terreno onde será construído até informações quanto a funcionalidade da estrutura o que facilita o quantitativo e checagem de interferências de projeto. A utilização da modelagem em BIM resulta em maior qualidade, menores imprevisto de execução e redução de tempo e de custo (EASTMAN ET AL; 2014).

Ao analisar obras de infraestrutura, a primeira análise a ser feita na região da obra é a topográfica e geomorfológica. Com esses estudos iniciais é possível determinar a qualidade do solo e o aproveitamento deste em áreas com necessidade de aterramento (MORAIS; FALCÃO; 2019).

Assim, este trabalho abordará a elaboração de mapas hipsométricos, na tomada de decisão quanto as áreas de corte e aterro e a metodologia utilizada na execução do empreendimento, utilizando a modelagem em BIM para quantificar os volumes e as principais zonas de terraplanagem.

\section{OBJETIVOS}

O trabalho terá como objetivo estudar a importância da elaboração de mapas hipsométricos para a tomada de decisões quanto ao tipo de métodos e determinação gráfica das zonas necessárias de terraplanagem.

\section{METODOLOGIA}

A Hipsometria refere-se a variação de altitude (Silveira et al.; 2013). Com base no Modelo Digital do Terreno (MDT), que segundo Silveira et al. (2013) permite cálculos associados ao relevo com rapidez e precisão, é projetada a terraplanagem da área analisada. $\mathrm{O}$ MDT de projeto é criado a partir de uma nuvem de pontos, no Software AutoCad Civil 3D, desenvolvido pela Autodesk. Segundo a desenvolvedora do software, Autodesk (2019), a superfície é criada a partir da interpolação dos vértices de triângulos criados entre pontos próximos. Assim sendo possível determinar a elevação de qualquer ponto dentro da superfície.

Ao analisar um terreno no município de Aquiraz no Ceará, foi feita uma análise no MDT de terreno natural, conforme Figura 01. Com o fim de obter o melhor terreno para os demais projetos no terreno e com o menor movimento de terra, chegou-se ao MDT de projeto, conforme Figura 02. 
Figura 1 - Modelo Digital de Terreno Natural

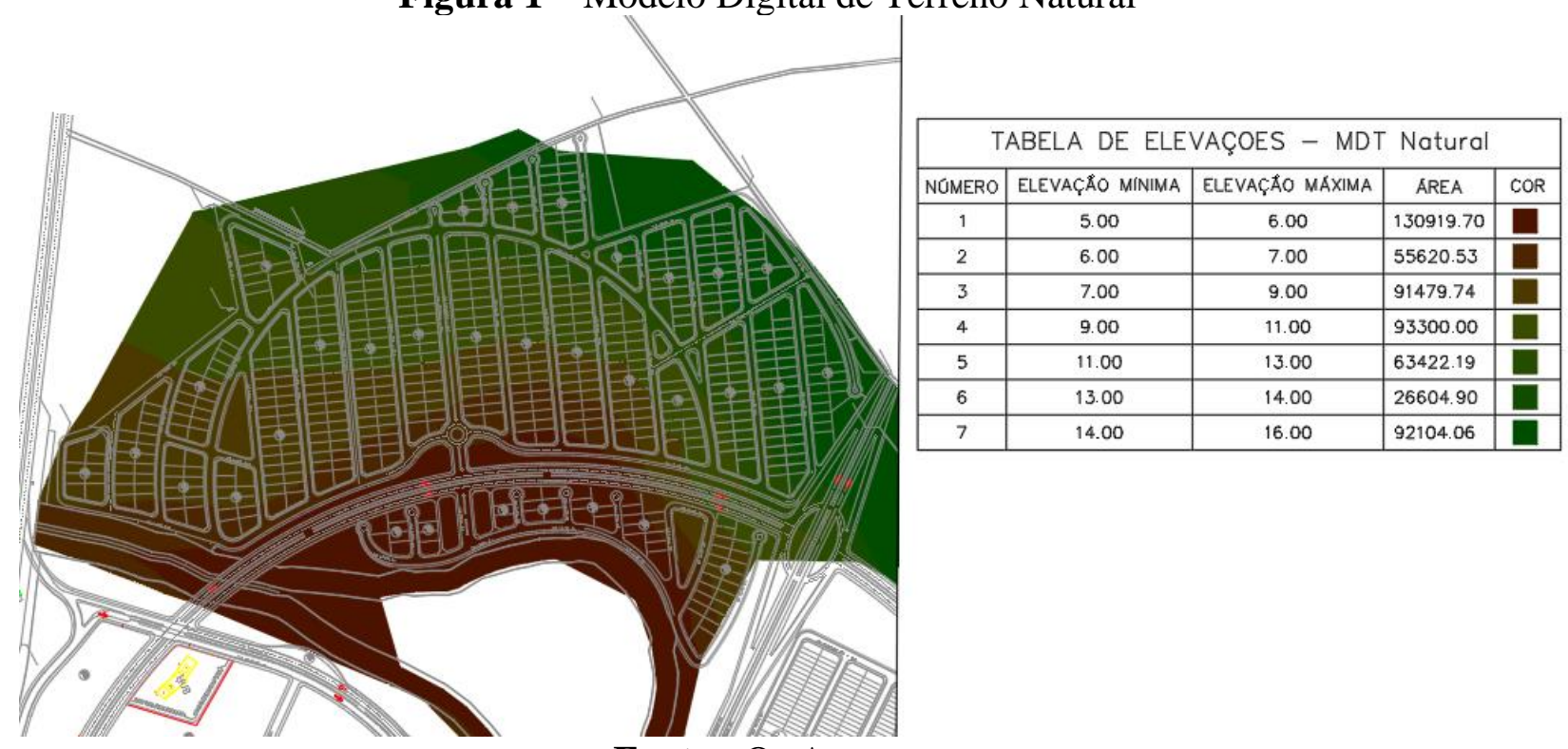

Fonte - Os Autores

Figura 2 - Modelo Digital de Terreno Projetado

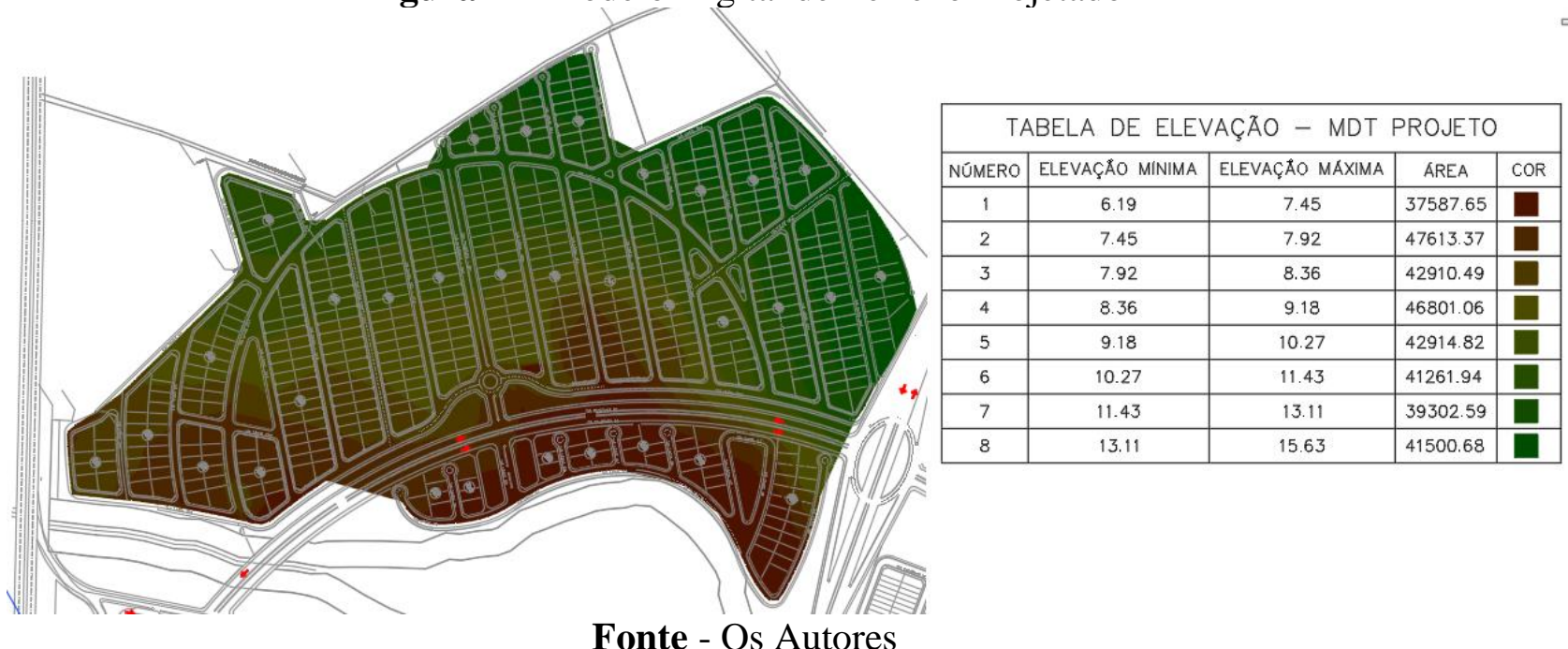

Com a diferença entre a cota de projeto e a cota de terreno natural é feita uma classificação, baseada nas áreas da triangulação da diferença entre as superfícies. Cada área é classificada em cores e limitada por meio de uma setorização baseada nas variações de terreno existente, conforme Figura 03.

Figura 3 - Dados de elevação extraído do Software

\begin{tabular}{|c|c|c|c|c|}
\hline \multicolumn{5}{|c|}{ TABELA DE ELEVAÇÕES } \\
\hline Número & Elevação mínima [m] Elevação máxima [m] & Área [m²] & Color \\
\hline 1 & -1.81 & -0.79 & 37204.87 & $\square$ \\
\hline 2 & -0.79 & -0.40 & 35677.01 & \\
\hline 3 & -0.40 & -0.11 & 39626.36 & $\square$ \\
\hline 4 & -0.11 & 0.00 & 36771.23 & \\
\hline 5 & 0.00 & 0.20 & 49737.26 & \\
\hline 6 & 0.20 & 0.78 & 49727.39 & \\
\hline 7 & 0.78 & 1.40 & 42180.87 & \\
\hline 8 & 1.40 & 2.41 & 49930.60 & \\
\hline
\end{tabular}


Fonte - Os Autores

Com a classificação dessas áreas é possível zonear o terreno analisado de acordo com a variação de cota entre a superfície base, o MDT de terreno natural, e a superfície de comparação, o MDT do terreno projetado.

\section{RESULTADOS}

Após análise da diferença de superfície é possível gerar um mapa hipsométrico das áreas de corte e aterro, conforme Figura 04. Na Figura 04 é observado o loteamento em Aquiraz, Ceará o qual foi classificado de acordo com a diferença de cota, onde o tom mais avermelhado representa corte e o tom mais verde representa aterro.

Figura 04 - Mapa Hipsométrico de um terreno em Aquiraz-CE

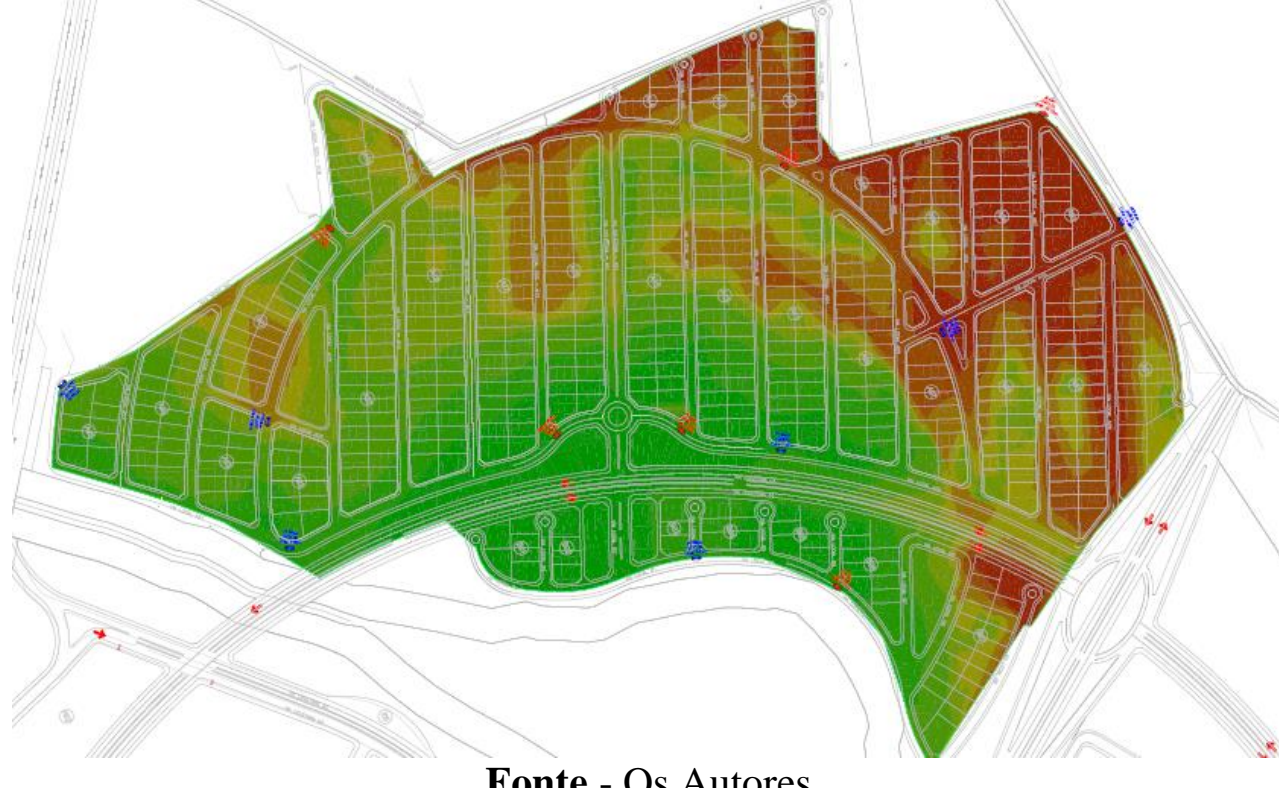

Essa classificação é imprescindível para a tomada, desde o método de movimento de terra a ser adotado na obra, até as áreas as quais deve haver maior movimentação e assim determinar as regiões onde devem iniciar a terraplanagem. Conforme analisado é ideal que as obras de terraplanagem se iniciem nas regiões mais a vermelho, conforme Figura 05, visto que nas zonas esverdeadas necessitam do material que será transportado das zonas avermelhas.

Figura 05 - Visualização hipsométrica de corte com triangulação

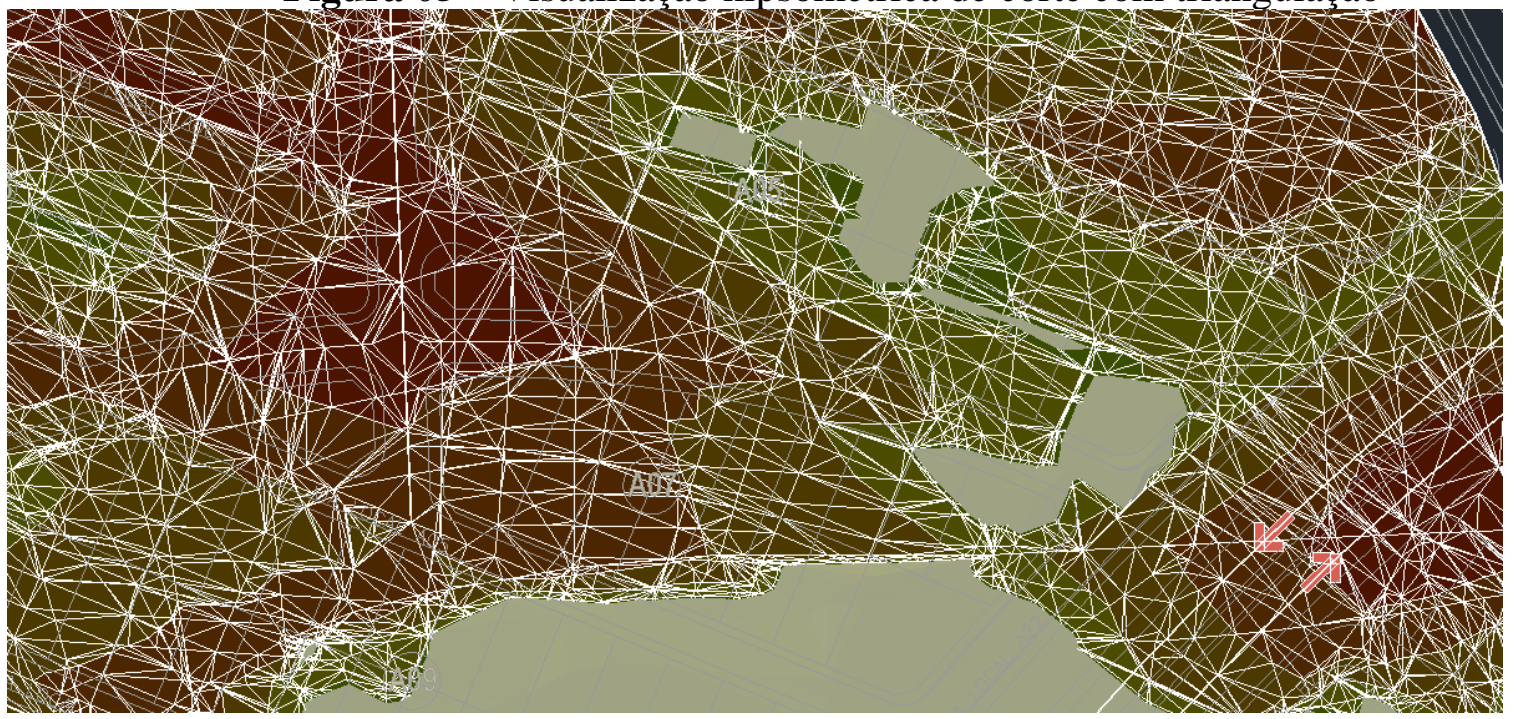


Fonte - Os Autores

\section{CONCLUSÃO}

Por conseguinte, a tomada de decisão nas obras de infraestrutura com ênfase nas obras de terraplanagem, pode ser aprovada posterior a análises de hipsometria de terreno natural e projeto. Dessa forma, a elaboração de mapas que evidencie a diferença entre esses terrenos se torna imprescindível na vivência do profissional de engenharia.

Assim, essa metodologia associada à software que utilizem o BIM como ferramenta, na fase de elaboração do projeto, otimiza o tempo de resposta com o planejamento de execução da obra. Com isso, há redução do volume de terra a ser transportado e compactadas e as zonas onde se iniciam os trabalhos de terraplanagem são definidas ainda na fase de projeto por meio da análise desses mapas.

Mapas como esses auxiliam os demais projetos de infraestrutura, já que trazem consigo informações, como o nível do terreno, que são primordiais para os demais projetos, por exemplo os de drenagem urbana, esgotamento sanitário e abastecimento de água.

\section{REFERÊNCIAS BIBLIOGRÁFICAS}

AUTODESK; Sobre a criação de uma superfície TIN; 2019. Disponivel em: https://knowledge.autodesk.com/pt-br/support/civil-3d/learn-explore/caas/CloudHelp/ cloudhelp/2019/PTB/Civil3D-UserGuide/files/GUID-D0FCED34-D68F-42D2-A6FB14C454CA57FA-htm.html Acesso em: 05 ago. 2020.

EASTMAN, C.; TEICHOLZ. P.; SACKS, R.; LISTON, K. Manual do BIM. Bookman, Porto Alegre, 2014.

MELO, E. DE A.; CALEGARIO, N.; DE MENDONÇA, A. R.; et al. MODELAGEM NÃO LINEAR DA RELAÇÃO HIPSOMÉTRICA E DO CRESCIMENTO DAS ÁRVORES DOMINANTES E CODOMINANTES DE Eucalyptus sp. MODELING. Ciencia Florestal, v. 27, n. 4, p. 1325-1338, 2017.

MORAIS, B. D. A.; FALCÃO, V. A. Otimização de equipamentos em obras de terraplanagem u lizando programação linear inteira. Revista Transportes, p. 134-144, 2019.

RODRIGUES, P. B. DE F.; MACHADO, R. L.; MENDES JÚNIOR, R.; ROMAGNOLI, L. D. S. C. Uma proposta de integração do modelo BIM ao sistema last planner. Ambiente Construído, v. 18, n. 4, p. 301-317, 2018.

SILVEIRA, C. T. DA; FIORI, A. P.; FERREIRA, A. M.; et al. EMPREGO DE ATRIBUTOS TOPOGRÁFICOS NO MAPEAMENTO DA SUSCEPTIBILIDADE A PROCESSOS GEOAMBIENTAIS NA BACIA DO RIO JACAREÍ, PARANÁ. , v. 25, n. 3, p. 623-639, 2013.

AUTODESK; Sobre a criação de uma superfície TIN; 2019. Disponivel em: https://knowledge.autodesk.com/pt-br/support/civil-3d/learn-explore/caas/CloudHelp/ cloudhelp/2019/PTB/Civil3D-UserGuide/files/GUID-D0FCED34-D68F-42D2-A6FB14C454CA57FA-htm.html Acesso em: 05 ago. 2020. 